\title{
Molecular imaging of coronary atherosclerosis; predictive of an acute myocardial infarction?
}

\author{
E. E. van der Wall
}

Published online: 28 November 2013

(C) The Author(s) 2013. This article is published with open access at Springerlink.com

Coronary atherosclerosis is a leading cause of cardiovascular morbidity and mortality worldwide. Plaque complications occur most commonly from plaque rupture, and also from plaque erosion and calcified nodule formation. Over the past years, advanced structural, metabolic and molecular imaging technologies have emerged and offer new windows into atherosclerosis pathophysiology [1-4]. Molecular imaging complements traditional structural plaque imaging through the use of targeted probes that identify specific molecules and/or biological processes in vivo [5]. Preclinical atherosclerosis molecular imaging has successfully identified nearly all established high-risk plaque characteristics including inflammation, thrombosis, neo-vessel formation, apoptosis, and haemorrhage. However, clinical translation of molecular imaging has been slow compared with the rapid growth within the field.

Currently, clinical atherosclerosis molecular imaging is dominated by non-invasive PET metabolism/inflammation plaque imaging with fluorine 18 (18F)-fluorodeoxyglucose (FDG). For the past two decades, 18F FDG has been the only fluorine-18 radioligand approved by the US Food and Drug Administration (FDA) for PET imaging. Coronary FDG PET is challenging, in part owing to the small size of coronary vessels and cardiac motion artefacts, but also because of the intense FDG uptake of the adjacent highly metabolic myocardium that obscures the coronary FDG signal. However, with dietary myocardial suppression protocols, detection of FDG PET signal in the left main and proximal coronary artery beds is sometimes achievable. Recently, non-invasive coronary FDG PET activity has been investigated in the stented culprit

E. E. van der Wall $(\bowtie)$

Interuniversity Cardiology Institute of the Netherlands

(ICIN) - Netherlands Heart Institute (NHI), P.O. Box 19258,

3501 DG Utrecht, the Netherlands

e-mail: ernst.van.der.wall@icin.nl lesions of 20 subjects with acute coronary syndromes (ACS) versus seven non-ACS subjects within 1 week of stent placement [6]. Using a predetermined FDG target to background ratio of 2.0 or greater to identify FDG-positive lesions, the ACS patients correlated positively with elevated stent FDG signal. However, coronary FDG signal was interpretable in only $50 \%$ of ACS patients, implying the need for further improvements in coronary FDG PET.

Apart from 18F FDG coronary imaging, clinically available approaches such as sodium $18 \mathrm{~F}$-fluoride $(\mathrm{NaF})$ for PET are emerging. Coronary calcification is a hallmark of advanced atheroma that can be detected with non-invasive $\mathrm{NaF}$ PET, which has been used for the past 3 decades to detect cancer bone metastases. As opposed to FDG, background myocardial uptake of $\mathrm{NaF}$ is negligible, and therefore $\mathrm{NaF}$ can determine coronary osteogenic activity. In a study by Dweck et al. from the University of Edinburgh [7], NaF PET molecular imaging identified sites of active coronary calcification non-invasively by PET and may be a new therapeutic target. The NaF signal was elevated in proportion to coronary CT calcium scores in 119 subjects, except in those with the highest calcium scores that may represent end-stage calcified plaques. From the same research group, Joshi et al. [8] recently (Lancet November 2013) studied coronary plaque imaging using two different radioactive tracers: $18 \mathrm{~F}-\mathrm{NaF}$ and 18F-FDG. The Scottish study included 40 patients with acute myocardial infarction (AMI) and 40 patients with stable angina. All patients underwent PET-CT, invasive coronary angiography, CT coronary angiography, and CT calcium scoring.

Among the 40 patients with AMI, 18F-NaF PET uptake was $34 \%$ higher in the culprit ruptured plaque compared with non-culprit plaques. Nearly all patients $(93 \%)$ had increased $18 \mathrm{~F}-\mathrm{NaF}$ uptake in the culprit plaque. With 18F-FDG, however, no differences in uptake between culprit and non-culprit plaques were observed. Among the 40 patients with stable angina, $45 \%$ had plaques with increased $18 \mathrm{~F}-\mathrm{NaF}$ uptake. 
These plaques were mostly non-obstructive at coronary angiography and contained more high-risk features on intravascular ultrasound compared with plaques without tracer uptake. Consequently, 18F-NaF PET may become an effective tool for predicting an AMI, which would have a great impact for patients with coronary atherosclerosis. Early detection of the atherosclerotic 'danger zones' could include immediate instalment of drugs such as statins or aspirin, drastic changes in lifestyle and even inserting stents into the affected coronary artery.

The use of 18F-NaF PET-CT offers the first non-invasive imaging method to identify and localise ruptured and highrisk coronary plaques. A next step is to show that increased $18 \mathrm{~F}-\mathrm{NaF}$ predicts future adverse clinical events. Further studies are therefore needed to evaluate whether the detection of risky plaques before rather than after an AMI has the potential to save lives. To summarise, the addition of newer molecular imaging tools such as NaF PET-CT will continue to strengthen our understanding of the in vivo biology of high-risk plaques [9, 10]. They are increasingly being translated into clinical use, and in combination with structural imaging they provide more comprehensive information to build risk-prediction tools. Ongoing efforts to improve non-invasive coronary imaging strategies must be pursued. Overall, with continued advances in molecular and structural atherosclerosis imaging, high-risk coronary atherosclerotic plaques will be brightened as never before. The recent study in the Lancet at least suggests that molecular imaging using PET-CT scanning may provide an answer in identifying 'ticking time bomb' patients at risk of an AMI.

Open Access This article is distributed under the terms of the Creative Commons Attribution License which permits any use, distribution, and reproduction in any medium, provided the original author(s) and the source are credited.

\section{References}

1. Fleg JL, Stone GW, Fayad ZA, et al. Detection of high-risk atherosclerotic plaque: report of the NHLBI Working Group on current status and future directions. JACC Cardiovasc Imaging. 2012;5:941-55.

2. van der Wall EE, Heidendal GA, den Hollander W, et al. Metabolic myocardial imaging with 123I-labeled heptadecanoic acid in patients with angina pectoris. Eur J Nucl Med. 1981;6: 391-6.

3. Knaapen P, de Haan S, Hoekstra OS, et al. Cardiac PET-CT: advanced hybrid imaging for the detection of coronary artery disease. Neth Heart J. 2010;18:90-8.

4. van der Wall EE, den Hollander W, Heidendal GA, et al. Dynamic myocardial scintigraphy with 123I-labeled free fatty acids in patients with myocardial infarction. Eur J Nucl Med. 1981;6:383-9.

5. Thomas G, Haraszti R. A new frontier in atherosclerotic coronary imaging. Lancet. 2013. doi:10.1016/S0140-6736(13) 61911-X.

6. Cheng VY, Slomka PJ, Le Meunier L, et al. Coronary arterial $18 \mathrm{~F}-\mathrm{FDG}$ uptake by fusion of PET and coronary CT angiography at sites of percutaneous stenting for acute myocardial infarction and stable coronary artery disease. J Nucl Med. 2012;53:575-83.

7. Dweck MR, Chow MW, Joshi NV, et al. Coronary arterial 18Fsodium fluoride uptake: a novel marker of plaque biology. J Am Coll Cardiol. 2012;59:1539-48.

8. Joshi NV, Vesey AT, Williams MC, et al. 18F-fluoride positron emission tomography for identification of ruptured and high-risk coronary atherosclerotic plaques: a prospective clinical trial. Lancet. 2013. doi:10.1016/S0140-6736(13)61754-7.

9. George RT. 18F-sodium fluoride positron emission tomography: an in vivo window into coronary atherosclerotic plaque biology. J Am Coll Cardiol. 2012;59:1549-50. doi:10.1016/j. jacc.2012.01.029.

10. Chen W, Dilsizian V. Targeted PET/CT imaging of vulnerable atherosclerotic plaques: microcalcification with sodium fluoride and inflammation with fluorodeoxyglucose. Curr Cardiol Rep. 2013;15: 364. doi:10.1007/s11886-013-0364-4. 